\title{
Recent Progress on the Molecular Mechanism of Quality Controls Induced by Ribosome Stalling
}

\author{
Ken Ikeuchi, Toshiaki Izawa and Toshifumi Inada* \\ Gene Regulation Laboratory, Graduate School of Pharmaceutical Sciences, Tohoku University, Sendai, Japan
}

Accurate gene expression is a prerequisite for all cellular processes. Cells actively promote correct protein folding, which prevents the accumulation of abnormal and nonfunctional proteins. Translation elongation is the fundamental step in gene expression to ensure cellular functions, and abnormal translation arrest is recognized and removed by the quality controls. Recent studies demonstrated that ribosome plays crucial roles as a hub for gene regulation and quality controls. Ribosome-interacting factors are critical for the quality control mechanisms responding to abnormal translation arrest by targeting its products for degradation. Aberrant mRNAs are produced by errors in mRNA maturation steps and cause aberrant translation and are eliminated by the quality

OPEN ACCESS

Edited by:

Akio Kanai,

Keio University, Japan

Reviewed by:

Hyouta Himeno,

Hirosaki University, Japan Yoshihiro Shimizu,

Riken, Japan

*Correspondence:

Toshifumi Inada

tinada@m.tohoku.ac.jp;

tinada@mail.pharm.tohoku.ac.jp

Specialty section:

This article was submitted to

$R N A$,

a section of the journal

Frontiers in Genetics

Received: 11 October 2018 Accepted: 22 December 2018 Published: 17 January 2019

Citation:

Ikeuchi K, Izawa T and Inada T (2019) Recent Progress on the Molecular Mechanism of Quality

Controls Induced by Ribosome

Stalling. Front. Genet. 9:743.

doi: 10.3389/fgene.2018.00743 control system. In this review, we focus on recent progress on two quality controls, Ribosome-associated Quality Control (RQC) and No-Go Decay (NGD), for abnormal translational elongation. These quality controls recognize aberrant ribosome stalling and induce rapid degradation of aberrant polypeptides and mRNAs thereby maintaining protein homeostasis and preventing the protein aggregation.

Keywords: ribosome, ribosome stalling, no-go mRNA decay, ribosome-associated quality control, ribosome ubiquitination

\section{INTRODUCTION}

Protein synthesis is a fundamental step of gene expression in all organisms. Translation elongation is perturbed by unique sequences, for instance poly-adenosine tract (Dimitrova et al., 2009), tandem rare codons such as yeast arginine CGA rare codon (Doma and Parker, 2006; Chen et al., 2010; Letzring et al., 2013), inhibitory di-codon pairs (Gamble et al., 2016), oxidized RNA (Simms et al., 2014) and robust higher order mRNA structures (Doma and Parker, 2006; Tsuboi et al., 2012), or by the severe cellular conditions including amino acid starvation (Guydosh and Green, 2014), tRNA deficiency (Ishimura et al., 2014), oxidative stress (Simms et al., 2014) and genetic mutations (LaRiviere et al., 2006). Ribosome stalling on the specific sites result in the perturbation of ribosome recycling as well as the production of aberrant truncated proteins. Cells have quality control systems to recognize ribosome stalling and eliminate the aberrant mRNAs and proteins. The stalled ribosome by the tandem CGA codons or KKK codon cluster is subjected to Ribosomeassociated Quality Control (RQC) that induces co-translational degradation of the arrest products (Dimitrova et al., 2009; Bengtson and Joazeiro, 2010; Brandman et al., 2012). RQC machinery is well conserved from yeast to human cells and related not only in cytosolic protein quality control but also in mitochondrial function, protein aggregation, and neurodegeneration (Wilson et al., 2007; Chu et al., 2009; Bengtson and Joazeiro, 2010; Brandman et al., 2012; Brandman and Hegde, 2016; 
Shao and Hegde, 2016; Garzia et al., 2017; Matsuo et al., 2017; Sitron et al., 2017; Sundaramoorthy et al., 2017; Juszkiewicz et al., 2018; Kuroha et al., 2018; Szadeczky-Kardoss et al., 2018; Verma et al., 2018; Zurita Rendon et al., 2018). No-go mRNA decay (NGD) is a eukaryotic mRNA quality control system and triggered by endonucleolytic cleavage in the vicinity of the stalled site followed by the exoribonucleolytic decay (van Hoof et al., 2002; Doma and Parker, 2006). In this review, we mainly describe the mechanism of RQC and NGD in yeast because it has been extensively investigated in yeast.

\section{RIBOSOME-ASSOCIATED QUALITY CONTROL}

\section{Ribosome Ubiquitination Is Required for the Subunit Dissociation in RQC}

Dissociation of stalled ribosomes into 40S and 60S subunits is an essential step to initiate RQC. Recent studies have demonstrated that ubiquitylation of stalled ribosomes triggers the subunit dissociation. An E3 ubiquitin ligase $\mathrm{Hel} 2$ in yeast and its mammalian homolog ZNF598 play a crucial role in this process (Brandman et al., 2012; Letzring et al., 2013; Saito et al., 2015; Garzia et al., 2017; Matsuo et al., 2017; Sitron et al., 2017; Sundaramoorthy et al., 2017; Juszkiewicz et al., 2018). A current model of RQC-trigger pathway induced by ribosome stalling is shown in Figure 1. Hel2 was initially identified as a Histone E3 ligase 2 required for cytosolic excess histone proteins (Singh et al., 2012). Hel2 recognizes the ribosomes stalled at the CGA rare codon cluster or poly(A) stretches on the mRNA sequences and mediates K63-linked poly-ubiquitination of ribosomal small subunit uS10 at K6 and K8 (Matsuo et al., 2017; Ikeuchi et al., 2019). The resulting ubiquitinated ribosomes are split into the subunits and then subjected to the downstream RQC pathway (Matsuo et al., 2017). ZNF598 in mammals also recognizes stalled ribosomes at the poly(A) stretches. ZNF598 is located in the head region of the $40 \mathrm{~S}$ subunit and ubiquitinates both uS10 (at K4 and K8) and eS10 (at K138 and K139) (Garzia et al., 2017; Sundaramoorthy et al., 2017; Juszkiewicz et al., 2018). Asc1 in yeast and its mammalian homolog RACK1 are also required for stall-mediated early RQC pathway (Kuroha et al., 2010; Letzring et al., 2013; Sitron et al., 2017). RACK1 is responsible for ZNF598-mediated ribosome ubiquitination in human cells (Sundaramoorthy et al., 2017; Juszkiewicz et al., 2018), and Asc1/RACK1 locates on the 40S head near the Hel2/ZNF598 target proteins, uS10, eS10, and uS3. Notably, Asc1 is not essential for non-stop RQC (Matsuda et al., 2014; Ikeuchi and Inada, 2016), because Asc1 is vital for stalling on poly(A) sequence but not for Dom34-mediated ribosome splitting at the $3^{\prime}$ end of the non-stop mRNA(Ikeuchi and Inada, 2016).

\section{A Disome as a Structural Unit for RQC}

Recent studies strongly suggest that a di-ribosome (disome) is a structural unit for RQC (Ikeuchi et al., 2019; Juszkiewicz et al., 2018). Hegde and co-workers demonstrated that ZNF598 preferentially ubiquitinates the disome with the mammalian in vitro translation system (Juszkiewicz et al., 2018). The collided di-ribosome is a minimal unit for RQC by ZNF598. The collided di-ribosome structure reveals a broad $40 \mathrm{~S}-40 \mathrm{~S}$ interface where the ubiquitination target of ZNF 598 is present (Juszkiewicz et al., 2018). It was proposed that the use of ribosomal collisions on behalf of stall makes it possible to adjust the degree of acceptable deceleration by the initiation rate for that mRNA (Juszkiewicz et al., 2018).

In yeast, CGACCG repeat induces RQC and NGD quality controls in vivo (Ikeuchi et al., 2019). The disomes formed by the CGACCG repeat are preferred as targets for Hel2-mediated uS10 ubiquitination over monosomes in vitro translation system (Ikeuchi et al., 2019). The Cryo-EM structure of the disome revealed that the leading ribosome is stalled in the classical POST-translocation state with an empty A-site and occupied Pand E-sites. The second ribosome is locked in an incomplete translocation step, and in a hybrid state with $\mathrm{A} / \mathrm{P}$ and $\mathrm{P} / \mathrm{E}-$ tRNAs. The interface between the leading and the colliding ribosomes is mainly formed by the small 40S subunit (Ikeuchi et al., 2019). The Cryo-EM analysis of Hel2-bound ribosome revealed that Hel2 preferentially binds to the rotated ribosome with the hybrid tRNAs (Matsuo et al., 2017). Therefore, it is possible that Hel2 binds to the colliding ribosome because it is the rotated form with the hybrid tRNAs. Importantly, the $40 \mathrm{~S}$ interribosomal contact interface brings all proteins targeted by Hel2 during quality control nearby. Moreover, both Asc1 (RACK1 in humans) molecules are in direct contact forming one of the inter-ribosomal interaction sites in a disome. It may represent the ideal substrate for Hel2, thereby specifically recognizing a prolonged translation stall to initiate RQC by its E3 ubiquitin ligase activity.

\section{The RQT Complex Has Potential to Dissociate the Subunit in RQC}

Recent studies have identified the RQC trigger (RQT) complex that is associated with translating ribosomes and essential for dissociation of stalled ribosomes (Matsuo et al., 2017). The RQT complex is composed of double RecA helicase domain-containing protein Slh1/Rqt2, CUE domain-containing ubiquitin-binding protein Cue3/Rqt3 and C2HC5-type zincfinger (ZnF) protein Ykr023w /Rqt4 (Matsuo et al., 2017; Sitron et al., 2017). The RQT function depends on the ATPase hydrolysis motif of Slh1/Rqt2 and moderately accelerated by the CUE domain of Cue3, indicating that the RQT complexmediated recognition of the ubiquitin on the ribosome promotes ribosome splitting. It remains elusive how the RQT complex acts to induce splitting of the stalled ribosome and how helicase activity of Slh1 works on the stalled complex. Additionally, the function of Rqt4 and the role of its unique $\mathrm{ZnF}$ domain are still undefined. In a mammal, ASCC3 has similarity to yeast Slh1/Rqt2 and is required for mammalian RQC induction (Matsuo et al., 2017). Human orthologs of Rqt3-4 remain to be clarified. It has been reported that ASCC3 forms ASC-1 complex (ASCC) with CUE domain containing protein ASCC2, RNA ligase-like protein ASCC1 and C2HC5-Znf protein TRIP4/ASC1 (Jung et al., 2002). Moreover, ASCC with demethylase ALKBH3 


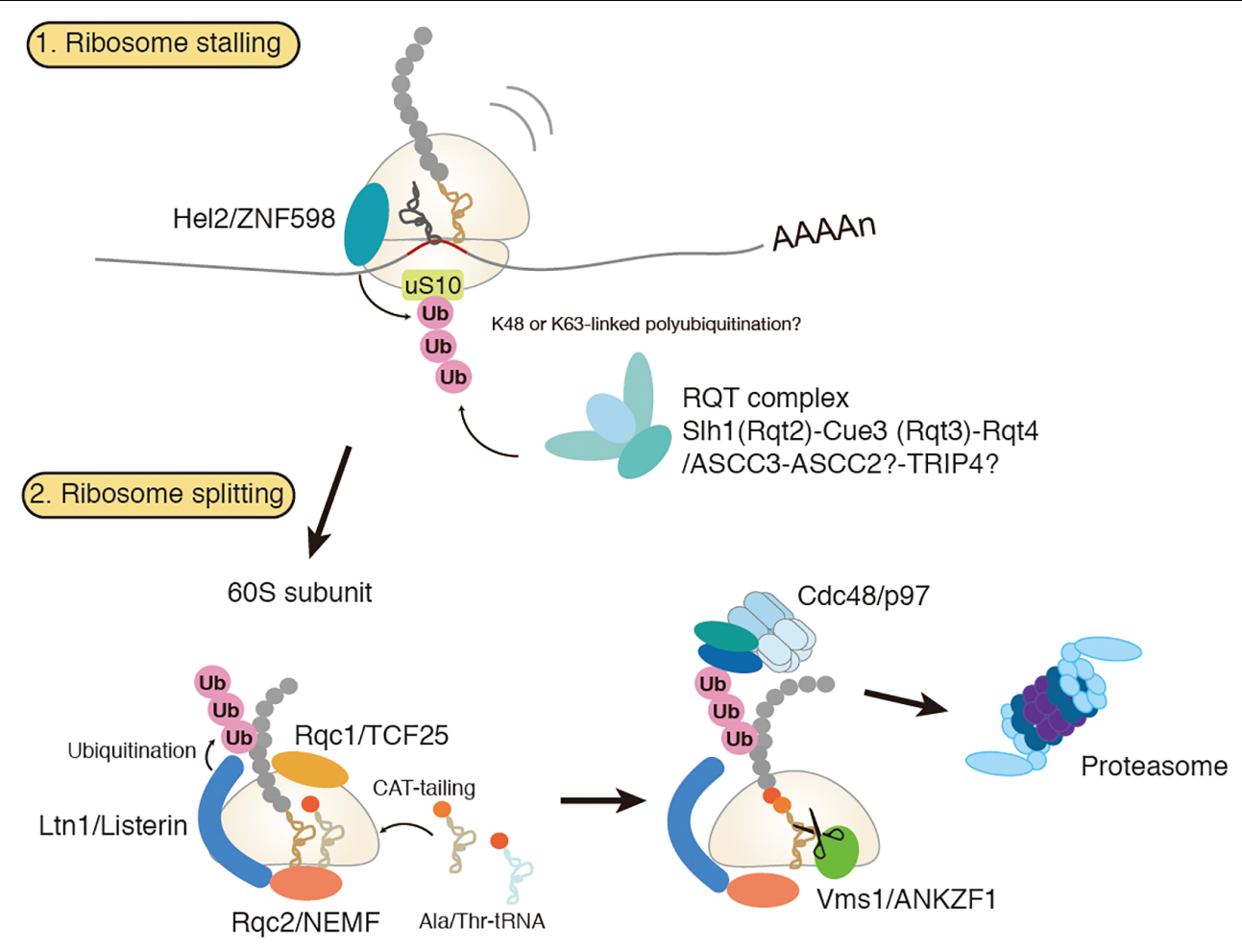

FIGURE 1 | A model for ribosome-associated protein quality control (RQC). Hel2/ZNF598 binds to the stalled ribosome and mediates ubiquitination of uS10 protein of $40 S$ ribosomal subunit. Subsequently, RQT complex, which is composed of Slh1/Rqc2, Cue3/Rqt3, and Rqt4, binds to the ubiquitylated ribosome to trigger splitting of ribosome and RQC. Stalled polypeptide on 60S ribosome is ubiquitylated by E3 ligase Ltn1 in concerted action with Rqc1. Rqc2 mediates elongation of stalled polypeptides by the C-terminal addition of multiple alanyl and threonyl residues (CAT-tailing). A ubiquitylated polypeptide is released by $\mathrm{Vms} 1$ and extracted by Cdc48 for proteasomal degradation.

forms complex to repair DNA alkylation in nuclear foci, and ASCC2 binds to K63-linked ubiquitin via its CUE domain, is critical for ASCC-ALKBH3 recruitment in the repairing foci (Brickner et al., 2017). At the moment, ASCC2 and TRIP4 are the potential mammalian orthologs of Cue3/Rqt3 and Rqt4, respectively, and might act as the mammalian RQTcomplex.

\section{Quality Control of Nascent Proteins on the 605 Subunit}

Stalling of ribosome can generate faulty proteins with cytotoxic properties. To prevent accumulation of such toxic proteins, cells have evolved the RQC complex that targets them for proteasomal degradation (Bengtson and Joazeiro, 2010; Brandman et al., 2012; Defenouillere et al., 2013; Verma et al., 2013). RQC complex consists of Ltn1, Rqc1, Rqc2, and Cdc48 (Listerin, TCF25, NEMF and p97 in a mammal, respectively). RQC complex is recruited to the $60 \mathrm{~S}$ subunit-peptidyl-tRNA complex after splitting of the stalled ribosome (Shao et al., 2013; Shao and Hegde, 2014). Rqc1 is also involved in this ubiquitination step, yet its function is little understood (Brandman et al., 2012; Defenouillere et al., 2013). Rqc2 facilitates recruitment of Ltn1 to the 60S subunit (Lyumkis et al., 2014; Shao et al., 2015). Rqc2 also mediates elongation of stalled nascent polypeptides on the $60 \mathrm{~S}$ subunit by the C-terminal addition of multiple alanyl and threonyl residues (CAT-tailing) in a template-free and 40S subunit independent manner (Shen et al., 2015). A recent study has shown that CAT-tailing acts as a fail-safe mechanism for efficient ubiquitination by Ltn1, by extracting the lysine residues sequestered in the ribosomal tunnel to the cytosol so that the lysine residues are accessible to Ltn1 (Kostova et al., 2017). Cdc48/p97, together with its co-factors Ufd1 and Npl4, acts in the downstream step to extract the nascent polypeptides for proteasomal degradation (Brandman et al., 2012; Defenouillere et al., 2013; Verma et al., 2013). An important question remaining to be solved is the mechanism for releasing the peptidyl-tRNA from the 60S subunit. Recent studies have identified Vms1 (ANKZF1 in a mammal) as a peptidyl-tRNA hydrolase in RQC. Vms1/ANKZF1 has a eukaryotic release factor 1 (eRF1) like domain with the conserved Gln residue that is supposed to catalyze peptidyl-tRNA hydrolysis in a similar way to the Gln residue of conserved GGQ motif in eRF1 (Verma et al., 2018; Zurita Rendon et al., 2018). However, the GGQ motif is not conserved, but is deviated to GSQ in yeast Vms1 and even to TAQ in human ANKZF1. More recently, ANKZF1 has been reported to function as a tRNA endonuclease rather than as a peptidyl-tRNA hydrolase in vitro (Kuroha et al., 2018). Future structural and biochemical studies will uncover the detailed mechanism of peptidyl-tRNA hydrolysis and tRNA cleavage by Vms1/ANKZF1. 
Failure of ubiquitination by a loss of Ltn 1 function causes accumulation of CAT-tailed proteins (Shen et al., 2015). Recently, several groups have reported that CAT-tailed proteins have a strong propensity to aggregate and cause proteotoxic stress in yeast (Choe et al., 2016; Defenouillere et al., 2016; Yonashiro et al., 2016; Izawa et al., 2017). They sequester multiple essential chaperones and form SDS-resistant aggregates, thereby interfering with general protein quality control pathways. CAT-tailed proteins have also reported to have an extremely toxic effect on mitochondria. The CAT-tailed mitochondrial proteins, synthesized in the cytosol but once imported into the mitochondria, sequester multiple essential chaperones, proteases and the components of translation machinery in the mitochondrial matrix, resulting in defective assembly of respiratory chain complexes and cell death (Izawa et al., 2017). In this context, Vms1 also acts as a key player to protect mitochondria from the toxic effect of CAT-tailed mitochondrial proteins. Vms1 antagonizes Rqc2, thereby preventing CATtailing and facilitating the release of stalled mitochondrial proteins to the downstream quality control network in the mitochondrial matrix (Izawa et al., 2017). Vms1 is particularly crucial for stalled mitochondrial proteins that cannot be ubiquitylated by Ltn1 due to the coupling of translation and protein import across the mitochondrial membranes. Since all the RQC components are conserved in eukaryotes including human, clearance of stalled proteins may be of general significance for cellular homeostasis. Indeed, the listerin hypomorphic mouse was shown to cause neurodegeneration (Chu et al., 2009). Further studies will clarify the roles of RQC in protein aggregation, mitochondrial dysfunction, and disease progression.

\section{NO-GO mRNA DECAY AND ROLES OF QUALITY CONTROL FACTORS}

No-go mRNA decay (NGD) is a cytosolic quality control system for mRNA induced by the ribosome stalling. NGD system is firstly discovered in Saccharomyces cerevisiae (Doma and Parker, 2006). NGD is conserved in fruit fly and plant, yet it has not been characterized in a mammal (Passos et al., 2009; SzadeczkyKardoss et al., 2018). The NGD is triggered by endonucleolytic cleavage of mRNA in the vicinity of the stalled ribosome, then the resulting the $5^{\prime}$ - and $3^{\prime}$-fragments are rapidly degraded by the exoribonucleolytic cleavages (Figure 2).

\section{Roles of Ribosome Collision and Ribosome Ubiquitination in NGD}

A recent study has proposed that ribosome collision is a critical trigger for mRNA cleavages in the initial step of NGD (Simms et al., 2017). In the study, accumulation of ribosomes on the mRNA has been observed as multiple cleavage sites which were distributed $43 \sim 300 \mathrm{nt}$ upstream of the stalling site with approximately $30 \mathrm{nt}$ periodicity likely due to the stacked ribosomes array. Similar situations that can be evidence for ribosome collision were observed on the GFP-Rz (hammerhead ribozyme) auto-cleaved truncated stop-codon-less reporter mRNA or the endogenous truncated mRNA under the dom34 deletion condition (Tsuboi et al., 2012; Guydosh and Green, 2014; Ikeuchi and Inada, 2016). Moreover, the collision of ribosome seems to be required for the cleavages of mRNA, as cleavage efficiency of mRNA was decreased with reduced initiation efficiency by using long $5^{\prime}$-UTR. Hel 2 was also proposed as a factor involved in NGD, and Hel2-mediated ubiquitination of ribosomal protein uS3 associates with ribosome collision. However, the requirement of $\mathrm{Hel} 2$ and ubiquitination of $\mathrm{US} 3$ in NGD is not manifested.

Although the mechanism of NGD has been studied using reporter genes, little is known about its endogenous targets. Ribosome footprinting is a powerful method for seeking cleavage sites of the endogenous targets (Guydosh et al., 2017). Recent sequencing-based strategy termed 5' hydroxyl RNA sequencing ( $5^{\prime} \mathrm{OH}$-seq) is a striking method to identify the $5^{\prime}$ ends of the cleaved intermediates (Peach et al., 2015; Ibrahim et al., 2018). Interestingly, NGD occurs under oxidative stress conditions (Simms et al., 2014), likely due to oxidized bases of mRNAs that interfere with base-pairing and cause aberrant translation elongation. The levels of K63-linked polyubiquitinated ribosomal proteins, translation factors, and various proteins were significantly increased upon oxidative stress by the down-regulation of deubiquitinating enzyme Ubp2 (Silva et al., 2015). These results strongly suggest a crucial role for K63-linked polyubiquitination of ribosomal proteins in NGD upon oxidative stress. To address the precise functions of K63linked polyubiquitination in NGD, the essential E3 ubiquitin ligase and its target sites should be identified.

\section{A Disome as a Unit for Hel2-Dependent $R Q C$ and NGD in Yeast}

Recent study by Beckmann and Inada labs demonstrated that the mRNA cleavage by the CGA rare codon cluster is dependent on Hel2-mediated K63-linked polyubiquitination (Ikeuchi et al., 2019). The determination of the cleavage sites by primer extension revealed that endonucleolytic cleavage of an NGD reporter mRNA occurs at sites within a disome unit consisting of the stalled ribosome and the following colliding ribosome. This minimal ribosome collision unit is required to couple NGD and RQC via Hel2. The cleavages in a disome require the ubiquitination of $\mathrm{uS} 10$ at lysine 6 th $(\mathrm{K} 6)$ or 8 th (K8) residues as well as the activity of the RQT component Slh1/Rqt2. Both Hel2-mediated ubiquitination of $\mathrm{uS} 10$ at K6 or K8 residues and Slh1/Rqt2 are essential for RQC, indicating that NGD and RQC are coupled via this ubiquitination event, and the NGD is referred to as the $\mathrm{NGD}^{\mathrm{RQC}+}$ (Figure 3). The determination of the cleavage sites in the RQC-defective mutants in which Hel2-dependent ubiquitination is defective or Slh1/Rqt2 deletion mutant revealed that the NGD pathway could be dissected into two interdependent branches. In this alternative NGD pathway, endonucleolytic mRNA cleavages occur upstream of the stalled disome (referred to as the $\mathrm{NGD}^{\mathrm{RQC}-}$; Figure 3). These cleavages require K63-linked polyubiquitination of ribosomal protein eS7. This polyubiquitination happens in a two-step mechanism, where the E3 ligase Not4 first monoubiquitinates eS7 which is followed 


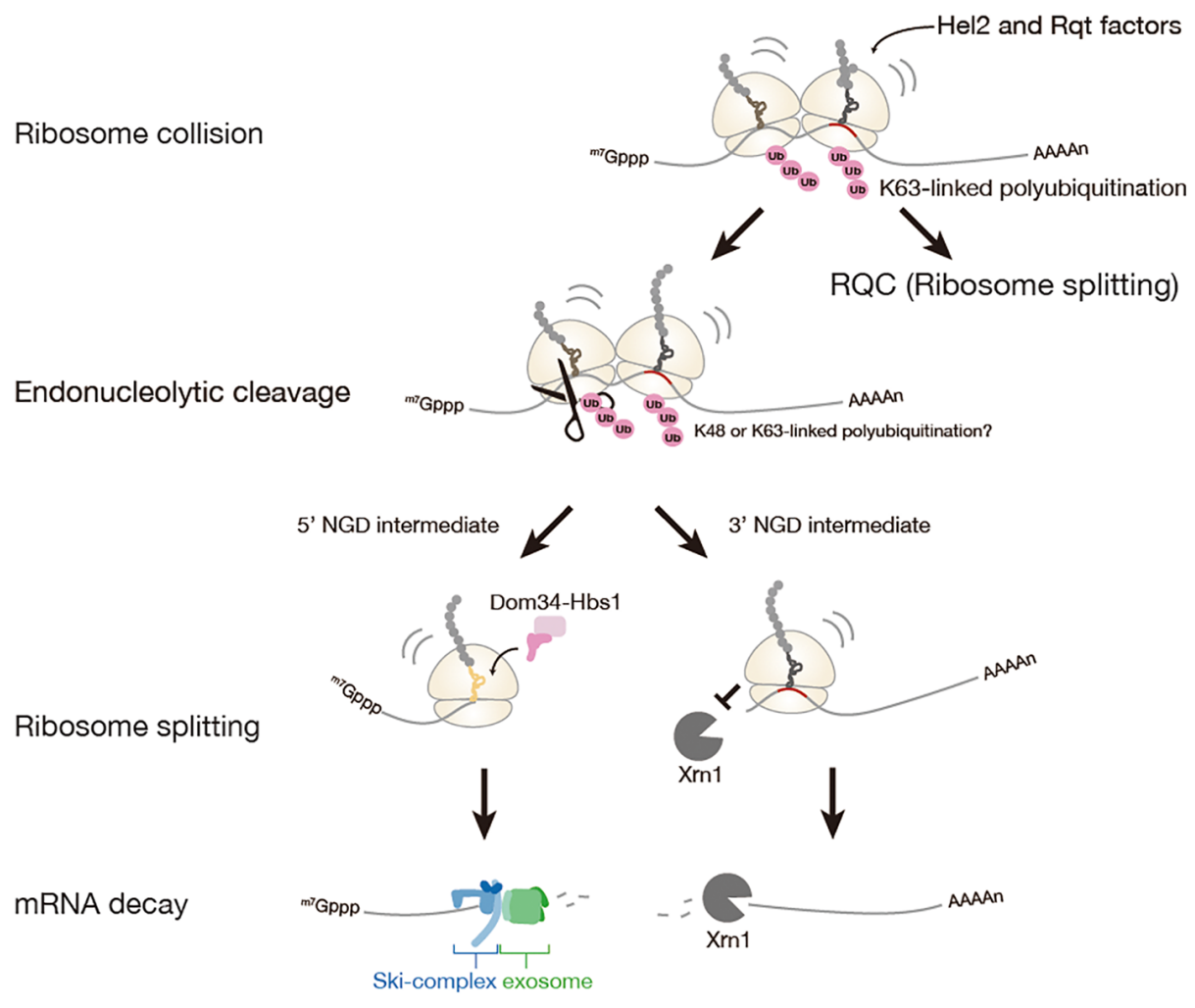

FIGURE 2 | No-Go mRNA decay triggered by ribosome stalling. A collision of stalled ribosomes leads to ubiquitination of 405 ribosomal subunit and endonucleolytic cleavage of mRNA. The stalled ribosome at the $3^{\prime}$-end of $5^{\prime}-\mathrm{NGD}$ intermediate is split by Dom34: Hbs 1 complex and the $5^{\prime}-\mathrm{NGD}$ intermediate is degraded by Ski complex and exosome. The stalled ribosome on the $3^{\prime}-\mathrm{NGD}$ intermediate may be dissociated or engaged in a restart of translation, and the $3^{\prime}$-NGD intermediate is degraded by Xrn1.
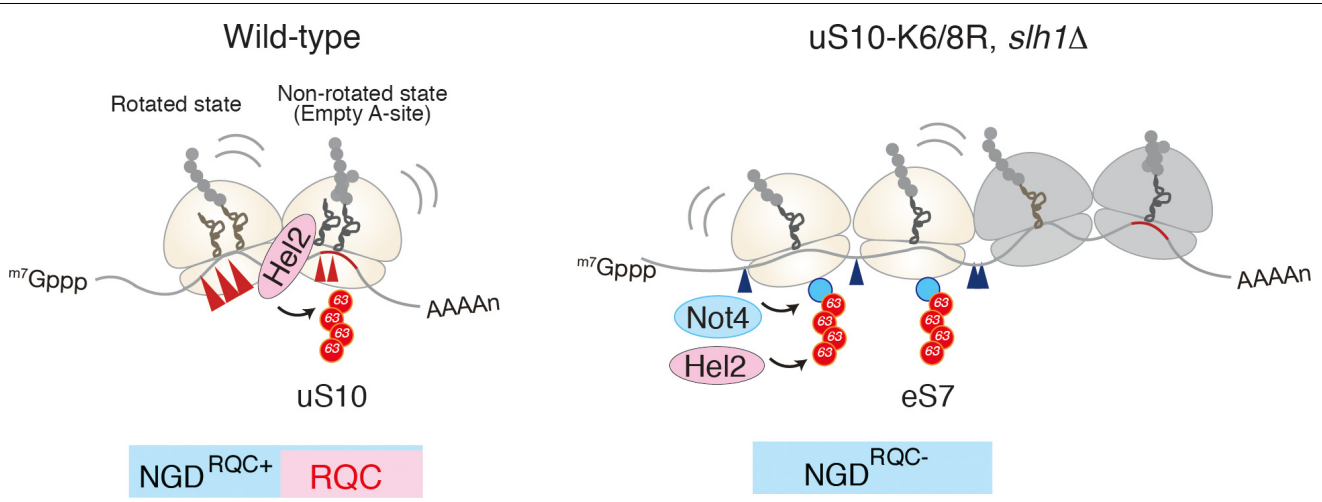

FIGURE 3 | A unique structural interface to induce Hel2-driven quality control pathways. Model for quality control pathways induced by R(CGN) 12 -mediated translation arrest (Ikeuchi et al., 2019). Hel2-mediated ribosome ubiquitination is required both for canonical NGD (NGDRQC+) and RQC coupled to the disome, and that RQC-uncoupled NGD outside the disome (NGD ${ }^{R Q C}-$ ) takes place in a Not4-mediated monoubiquitination dependent manner. The arrowheads indicate the endonucleolytic cleavages sites in NGD. The red line indicates the rare codon cluster. Left: the RQC pathway is intact, the leading ribosome that is stalled by the arrest sequence undergoes RQC. The uS10 ubiquitination and SIh1/Rqt2-dependent subunit dissociation induce the endonucleolytic cleavages in the disome. Right: In the absence of uS10 ubiquitination and Rqt2, RQC in the first ribosome, as well as NGD in the disome, are eliminated. RQC-uncoupled NGD ${ }^{R Q C}-$ takes place upstream of the disome. The figure concept has been reproduced from the original article (Ikeuchi et al., 2019).

by Hel2-mediated polyubiquitination. Finally, it was proposed that a dual role of Hel2 leading to two distinct NGD pathways, which require specific ubiquitination events on the stalled disome (Figure 3).
It is still unknown whether ribosome collision itself is essential for ribosome splitting and endoribonucleolytic cleavage, resulting in nascent peptide degradation and mRNA degradation. Hel2mediated uS10 ubiquitination is required for RQC and NGD 
in a disome, and the disomes are preferred as targets for Hel2-mediated uS10 ubiquitination over monosomes. This raises the question of whether the endoribonucleolytic cleavage is required for RQC, which is an essential question to understand the mechanism of the coupled quality controls by ribosome stalling.

\section{PERSPECTIVES}

Recent studies have clarified the novel molecular mechanisms how quality controls systems recognize stalling ribosome and eliminate aberrant products. However, many questions should be addressed including the relation between RQC and NGD, the roles of RQC factors in NGD. Future experiments and analyses will uncover the molecular mechanisms and

\section{REFERENCES}

Bengtson, M. H., and Joazeiro, C. A. (2010). Role of a ribosome-associated E3 ubiquitin ligase in protein quality control. Nature 467, 470-473. doi: 10.1038/ nature09371

Brandman, O., and Hegde, R. S. (2016). Ribosome-associated protein quality control. Nat. Struct. Mol. Biol. 23, 7-15. doi: 10.1038/nsmb.3147

Brandman, O., Stewart-Ornstein, J., Wong, D., Larson, A., Williams, C. C., Li, G. W., et al. (2012). A ribosome-bound quality control complex triggers degradation of nascent peptides and signals translation stress. Cell 151, 1042-1054. doi: 10.1016/j.cell.2012.10.044

Brickner, J. R., Soll, J. M., Lombardi, P. M., Vagbo, C. B., Mudge, M. C., Oyeniran, C., et al. (2017). A ubiquitin-dependent signalling axis specific for ALKBH-mediated DNA dealkylation repair. Nature 551, 389-393. doi: 10.1038/ nature 24484

Chen, L., Muhlrad, D., Hauryliuk, V., Cheng, Z., Lim, M. K., Shyp, V., et al. (2010). Structure of the Dom34-Hbs1 complex and implications for no-go decay. Nat. Struct. Mol. Biol. 17, 1233-1240. doi: 10.1038/nsmb.1922

Choe, Y. J., Park, S. H., Hassemer, T., Korner, R., Vincenz-Donnelly, L., HayerHartl, M., et al. (2016). Failure of RQC machinery causes protein aggregation and proteotoxic stress. Nature 531, 191-195. doi: 10.1038/nature16973

Chu, J., Hong, N. A., Masuda, C. A., Jenkins, B. V., Nelms, K. A., Goodnow, C. C., et al. (2009). A mouse forward genetics screen identifies LISTERIN as an E3 ubiquitin ligase involved in neurodegeneration. Proc. Natl. Acad. Sci. U.S.A. 106, 2097-2103. doi: 10.1073/pnas.0812819106

Defenouillere, Q., Yao, Y., Mouaikel, J., Namane, A., Galopier, A., Decourty, L., et al. (2013). Cdc48-associated complex bound to $60 \mathrm{~S}$ particles is required for the clearance of aberrant translation products. Proc. Natl. Acad. Sci. U.S.A. 110, 5046-5051. doi: 10.1073/pnas.1221724110

Defenouillere, Q., Zhang, E., Namane, A., Mouaikel, J., Jacquier, A., and FromontRacine, M. (2016). Rqc1 and Ltn1 prevent C-terminal alanine-threonine tail (CAT-tail)-induced protein aggregation by efficient recruitment of Cdc48 on stalled 60S subunits. J. Biol. Chem. 291, 12245-12253. doi: 10.1074/jbc.M116. 722264

Dimitrova, L. N., Kuroha, K., Tatematsu, T., and Inada, T. (2009). Nascent peptidedependent translation arrest leads to Not4p-mediated protein degradation by the proteasome. J. Biol. Chem. 284, 10343-10352. doi: 10.1074/jbc.M808840200

Doma, M. K., and Parker, R. (2006). Endonucleolytic cleavage of eukaryotic mRNAs with stalls in translation elongation. Nature 440, 561-564. doi: 10.1038/ nature 04530

Gamble, C. E., Brule, C. E., Dean, K. M., Fields, S., and Grayhack, E. J. (2016). Adjacent codons act in concert to modulate translation efficiency in yeast. Cell 166, 679-690. doi: 10.1016/j.cell.2016.05.070

Garzia, A., Jafarnejad, S. M., Meyer, C., Chapat, C., Gogakos, T., Morozov, P., et al. (2017). The E3 ubiquitin ligase and RNA-binding protein ZNF598 orchestrates ribosome quality control of premature polyadenylated mRNAs. Nat. Commun. 8:16056. doi: $10.1038 /$ ncomms 16056 biological functions of quality controls induced by ribosome stalling.

\section{AUTHOR CONTRIBUTIONS}

All authors listed have made a substantial, direct and intellectual contribution to the work, and approved it for publication.

\section{FUNDING}

TIn was supported by a Grant-in-Aid for Scientific Research (KAKENHI) from the Japan Society for the Promotion of Science (Grant No. 26116003), and by Research Grants in the Natural Sciences from the Takeda Foundation.

Guydosh, N. R., and Green, R. (2014). Dom34 rescues ribosomes in 3' untranslated regions. Cell 156, 950-962. doi: 10.1016/j.cell.2014.02.006

Guydosh, N. R., Kimmig, P., Walter, P., and Green, R. (2017). Regulated Ire1dependent mRNA decay requires no-go mRNA degradation to maintain endoplasmic reticulum homeostasis in S. pombe. Elife 6:e29216. doi: 10.7554/ eLife. 29216

Ibrahim, F., Maragkakis, M., Alexiou, P., and Mourelatos, Z. (2018). Ribothrypsis, a novel process of canonical mRNA decay, mediates ribosome-phased mRNA endonucleolysis. Nat. Struct. Mol. Biol. 25, 302-310. doi: 10.1038/s41594-0180042-8

Ikeuchi, K., and Inada, T. (2016). Ribosome-associated Asc1/RACK1 is required for endonucleolytic cleavage induced by stalled ribosome at the 3' end of nonstop mRNA. Sci. Rep. 6:28234. doi: 10.1038/srep28234

Ikeuchi, K., Tesina, P., Matsuo, Y., Sugiyama, T., Cheng, J., Saeki, Y., et al. (2019). Collided ribosomes form a unique structural interface to induce Hel2-driven quality control pathways. EMBO J. e100276. doi: 10.15252/embj.2018100276 [Epub ahead of print].

Ishimura, R., Nagy, G., Dotu, I., Zhou, H., Yang, X. L., Schimmel, P., et al. (2014). RNA function. Ribosome stalling induced by mutation of a CNS-specific tRNA causes neurodegeneration. Science 345, 455-459. doi: 10.1126/science.124 9749

Izawa, T., Park, S. H., Zhao, L., Hartl, F. U., and Neupert, W. (2017). Cytosolic protein Vms1 links ribosome quality control to mitochondrial and cellular homeostasis. Cell 171, 890.e18-903.e18. doi: 10.1016/j.cell.2017. 10.002

Jung, D. J., Sung, H. S., Goo, Y. W., Lee, H. M., Park, O. K., Jung, S. Y., et al. (2002). Novel transcription coactivator complex containing activating signal cointegrator 1. Mol. Cell. Biol. 22, 5203-5211. doi: 10.1128/MCB.22.14.52035211.2002

Juszkiewicz, S., Chandrasekaran, V., Lin, Z., Kraatz, S., Ramakrishnan, V., and Hegde, R. S. (2018). ZNF598 is a quality control sensor of collided ribosomes. Mol. Cell. 72, 469.e7-481.e7. doi: 10.1016/j.molcel.2018.08.037

Kostova, K. K., Hickey, K. L., Osuna, B. A., Hussmann, J. A., Frost, A., Weinberg, D. E., et al. (2017). CAT-tailing as a fail-safe mechanism for efficient degradation of stalled nascent polypeptides. Science 357, 414-417. doi: 10.1126/science. aam7787

Kuroha, K., Akamatsu, M., Dimitrova, L., Ito, T., Kato, Y., Shirahige, K., et al. (2010). Receptor for activated C kinase 1 stimulates nascent polypeptidedependent translation arrest. EMBO Rep. 11, 956-961. doi: 10.1038/embor. 2010.169

Kuroha, K., Zinoviev, A., Hellen, C. U. T., and Pestova, T. V. (2018). Release of ubiquitinated and non-ubiquitinated nascent chains from stalled mammalian ribosomal complexes by ANKZF1 and Ptrh1. Mol. Cell 72, 286.e8-302.e8. doi: 10.1016/j.molcel.2018.08.022

LaRiviere, F. J., Cole, S. E., Ferullo, D. J., and Moore, M. J. (2006). A late-acting quality control process for mature eukaryotic rRNAs. Mol. Cell 24, 619-626. doi: 10.1016/j.molcel.2006.10.008 
Letzring, D. P., Wolf, A. S., Brule, C. E., and Grayhack, E. J. (2013). Translation of CGA codon repeats in yeast involves quality control components and ribosomal protein L1. RNA 19, 1208-1217. doi: 10.1261/rna.039446.113

Lyumkis, D., Oliveira dos Passos, D., Tahara, E. B., Webb, K., Bennett, E. J., Vinterbo, S., et al. (2014). Structural basis for translational surveillance by the large ribosomal subunit-associated protein quality control complex. Proc. Natl. Acad. Sci. U.S.A. 111, 15981-15986. doi: 10.1073/pnas.1413882111

Matsuda, R., Ikeuchi, K., Nomura, S., and Inada, T. (2014). Protein quality control systems associated with no-go and nonstop mRNA surveillance in yeast. Genes Cells 19, 1-12. doi: 10.1111/gtc.12106

Matsuo, Y., Ikeuchi, K., Saeki, Y., Iwasaki, S., Schmidt, C., Udagawa, T., et al. (2017). Ubiquitination of stalled ribosome triggers ribosome-associated quality control. Nat. Commun. 8:159. doi: 10.1038/s41467-017-00188-1

Passos, D. O., Doma, M. K., Shoemaker, C. J., Muhlrad, D., Green, R., Weissman, J., et al. (2009). Analysis of Dom 34 and its function in no-go decay. Mol. Biol. Cell 20, 3025-3032. doi: 10.1091/mbc.E09-01-0028

Peach, S. E., York, K., and Hesselberth, J. R. (2015). Global analysis of RNA cleavage by 5'-hydroxyl RNA sequencing. Nucleic Acids Res. 43, e108. doi: 10.1093/nar/ gkv536

Saito, K., Horikawa, W., and Ito, K. (2015). Inhibiting K63 polyubiquitination abolishes no-go type stalled translation surveillance in Saccharomyces cerevisiae. PLoS Genet. 11:e1005197. doi: 10.1371/journal.pgen.1005197

Shao, S., Brown, A., Santhanam, B., and Hegde, R. S. (2015). Structure and assembly pathway of the ribosome quality control complex. Mol. Cell 57, 433-444. doi: 10.1016/j.molcel.2014.12.015

Shao, S., and Hegde, R. S. (2014). Reconstitution of a minimal ribosomeassociated ubiquitination pathway with purified factors. Mol. Cell 55, 880-890. doi: 10.1016/j.molcel.2014.07.006

Shao, S., and Hegde, R. S. (2016). target selection during protein quality control. Trends Biochem. Sci. 41, 124-137. doi: 10.1016/j.tibs.2015.10.007

Shao, S., von der Malsburg, K., and Hegde, R. S. (2013). Listerin-dependent nascent protein ubiquitination relies on ribosome subunit dissociation. Mol. Cell 50, 637-648. doi: 10.1016/j.molcel.2013.04.015

Shen, P. S., Park, J., Qin, Y., Li, X., Parsawar, K., Larson, M. H., et al. (2015). Protein synthesis. Rqc2p and $60 \mathrm{~S}$ ribosomal subunits mediate mRNA-independent elongation of nascent chains. Science 347, 75-78. doi: 10.1126/science.125 9724

Silva, G. M., Finley, D., and Vogel, C. (2015). K63 polyubiquitination is a new modulator of the oxidative stress response. Nat. Struct. Mol. Biol. 22, 116-123. doi: $10.1038 / \mathrm{nsmb} .2955$

Simms, C. L., Hudson, B. H., Mosior, J. W., Rangwala, A. S., and Zaher, H. S. (2014). An active role for the ribosome in determining the fate of oxidized mRNA. Cell Rep. 9, 1256-1264. doi: 10.1016/j.celrep.2014.10.042

Simms, C. L., Yan, L. L., and Zaher, H. S. (2017). Ribosome collision is critical for quality control during no-go decay. Mol. Cell 68, 361.e5-373.e5. doi: 10.1016/j. molcel.2017.08.019

Singh, R. K., Gonzalez, M., Kabbaj, M. H., and Gunjan, A. (2012). Novel E3 ubiquitin ligases that regulate histone protein levels in the budding yeast saccharomyces cerevisiae. PLoS One 7:e36295. doi: 10.1371/journal.pone. 0036295

Sitron, C. S., Park, J. H., and Brandman, O. (2017). Asc1, Hel2, and Slh1 couple translation arrest to nascent chain degradation. RNA 23, 798-810. doi: 10.1261/ rna.060897.117

Sundaramoorthy, E., Leonard, M., Mak, R., Liao, J., Fulzele, A., and Bennett, E. J. (2017). ZNF598 and RACK1 regulate mammalian ribosome-associated quality control function by mediating regulatory $40 \mathrm{~S}$ ribosomal ubiquitylation. Mol. Cell 65, 751.e4-760.e4. doi: 10.1016/j.molcel.2016.12.026

Szadeczky-Kardoss, I., Gal, L., Auber, A., Taller, J., and Silhavy, D. (2018). The Nogo decay system degrades plant mRNAs that contain a long a-stretch in the coding region. Plant Sci. 275, 19-27. doi: 10.1016/j.plantsci.2018.07.008

Tsuboi, T., Kuroha, K., Kudo, K., Makino, S., Inoue, E., Kashima, I., et al. (2012). Dom34:hbs1 plays a general role in quality-control systems by dissociation of a stalled ribosome at the 3' end of aberrant mRNA. Mol. Cell 46, 518-529. doi: 10.1016/j.molcel.2012.03.013

van Hoof, A., Frischmeyer, P. A., Dietz, H. C., and Parker, R. (2002). Exosomemediated recognition and degradation of mRNAs lacking a termination codon. Science 295, 2262-2264. doi: 10.1126/science.1067272

Verma, R., Oania, R. S., Kolawa, N. J., and Deshaies, R. J. (2013). Cdc48/p97 promotes degradation of aberrant nascent polypeptides bound to the ribosome. Elife 2:e00308. doi: 10.7554/eLife.00308

Verma, R., Reichermeier, K. M., Burroughs, A. M., Oania, R. S., Reitsma, J. M., Aravind, L., et al. (2018). Vms1 and ANKZF1 peptidyl-tRNA hydrolases release nascent chains from stalled ribosomes. Nature 557, 446-451. doi: 10.1038/ s41586-018-0022-5

Wilson, M. A., Meaux, S., and van Hoof, A. (2007). A genomic screen in yeast reveals novel aspects of nonstop mRNA metabolism. Genetics 177, 773-784. doi: 10.1534/genetics.107.073205

Yonashiro, R., Tahara, E. B., Bengtson, M. H., Khokhrina, M., Lorenz, H., Chen, K. C., et al. (2016). The Rqc2/Tae2 subunit of the ribosome-associated quality control (RQC) complex marks ribosome-stalled nascent polypeptide chains for aggregation. Elife 5:e11794. doi: 10.7554/eLife.11794

Zurita Rendon, O., Fredrickson, E. K., Howard, C. J., Van Vranken, J., Fogarty, S., Tolley, N. D., et al. (2018). Vmslp is a release factor for the ribosomeassociated quality control complex. Nat. Commun. 9:2197. doi: 10.1038/s41467018-04564-3

Conflict of Interest Statement: The authors declare that the research was conducted in the absence of any commercial or financial relationships that could be construed as a potential conflict of interest.

Copyright (C) 2019 Ikeuchi, Izawa and Inada. This is an open-access article distributed under the terms of the Creative Commons Attribution License (CC BY). The use, distribution or reproduction in other forums is permitted, provided the original author(s) and the copyright owner(s) are credited and that the original publication in this journal is cited, in accordance with accepted academic practice. No use, distribution or reproduction is permitted which does not comply with these terms. 